\title{
Kinematic Alignment Total Knee Replacement with Personalized Instruments
}

\author{
William G. Blakeney and Pascal-André Vendittoli
}

\section{Key Points}

- Performing a kinematically aligned (KA) TKA requires accurate planning of resections and precise tools to achieve the set goals.

- CT-based patient-specific instrumentation (PSI) is our preferred method for performing KA TKA implantation.

- The restricted kinematic alignment protocol (rKA) has been developed as an alternative solution to the "true" KA technique in situations of patients with atypical knee anatomy.

- The rKA protocol limits the femoral and tibial prosthesis coronal alignment to within $\pm 5^{\circ}$ of neutral, with the overall combined lower limb coronal orientation within $\pm 3^{\circ}$ of neutral.

Electronic Supplementary Material The online version of this chapter (https://doi.org/10.1007/978-3-030-242435_25) contains supplementary material, which is available to authorized users.

W. G. Blakeney

Department of Surgery, CIUSSS-de-L'Est-de-L'Ilede-Montréal, Hôpital Maisonneuve Rosemont, Montréal, QC, Canada

Department of Surgery, Albany Health Campus, Albany, WA, Australia

P.-A. Vendittoli $(\bowtie)$

Department of Surgery, CIUSSS-de-L'Est-de-L'Ilede-Montréal, Hôpital Maisonneuve Rosemont, Montréal, QC, Canada

Department of Surgery, Université de Montréal, Montréal, QC, Canada
- PSI allows for pre-operative planning and fine-tuning adjustments.

- Compared to standard instruments, computer navigation or robotic surgery, PSI results in a shorter operating time and decreased instrumentation.

- PSI is a simple, standardised solution for a patient-specific rKA protocol in TKA, with many benefits to the surgeon and patient.

\subsection{Personalized Instrumentation to Reproduce Patients' Specific Anatomy}

There is a very wide variation in patients' knee anatomy. The precise restoration of this anatomy during total knee arthroplasty (TKA) may improve knee balance, clinical function and patient satisfaction. In the early ages of TKA, implant sizes and surgical precision were limited. The amount of deviation from a patient's anatomy that may impact on clinical results is not clear. However, in the era of personalized joint replacement, we believe that a precision of within $2 \mathrm{~mm}$ or $2^{\circ}$ should be the goal. Performing a kinematically aligned (KA) TKA requires accurate planning of resections and precise tools to achieve the set goals. Patient-specific instrumentation is a very attractive solution. These patient-specific instruments (PSI) are constructed based upon 
preoperative planning using either tomographic or magnetic resonance imaging. 3D models of the patient's knee, hip and ankle are reconstructed and anatomical landmarks are identified to set the parameters of tibia and femur resections according to the surgeon's preferences. Intraoperatively, the custom guides or PSI is applied to the bone surfaces to guide resections. The PSI defines both the implant orientation and size.

Generally, CT-based imaging is preferred for KA TKA, as measurement of the patient's bony anatomy is required to measure the constitutional knee alignment of the patient. The majority of patients undergoing TKA have minimal bone loss with most of the articular surface wear being cartilaginous. Aligning the resections to the bony anatomy of the femur and tibia, accounting for an equal cartilage layer medial and lateral, allows restoration of the native joint line and alignment. When bone loss is present, however, this should be considered when planning resection planes. A metaanalysis compared the accuracy of MRI- and CT-based systems for PSI [1]. They reported the incidence of outliers greater than $3^{\circ}$ was $12.5 \%$ for CT-based systems vs. $16.9 \%$ for MRI-based systems, though this difference was not statistically significant.

The accuracy of PSI has been assessed by numerous studies and meta-analyses [2-10]. Three meta-analyses reported improved coronal femoral alignment with PSI compared to conventional instrumentation [6-8]. However, four other meta-analyses failed to detect a significant difference [2-5]. The tibial coronal alignment was demonstrated to favour conventional instrumentation over PSI in four meta-analyses $[2,4,5,8]$, whereas three did not detect any significant differences $[3,6,7]$. No significant difference was detected in femoral sagittal alignment in any of the meta-analyses. Four studies found an increased risk of tibial sagittal plane malalignment with PSI [2, 4, 5, 8]. One study found improved rotational alignment with PSI [10]. These studies all looked at the accuracy of a mechanical alignment (MA) protocol. The accuracy of PSI for a KA technique is likely to replicate these results.

\subsection{Restricted Kinematic Alignment Protocol and Personalized Instrumentation}

We developed and have used clinically, since 2011, a restricted KA protocol (rKA, see Chap. 17) [11]. The PSI method described (MyKnee ${ }^{\circledR}$, Medacta International SA, Castel San Pietro, Switzerland) is our preferred method for performing rKA TKA implantation. Pre-operative CT scans according to the standardised MyKnee ${ }^{\circledR}$ protocol are taken. Cutting blocks and 3D bone models of the knee are then produced according to the preferences of the surgeon. The rKA protocol aims to reproduce the patient's constitutional knee anatomy within a defined safe range [11, 12]. The rKA technique limits the femoral and tibial prosthesis coronal alignment to within $\pm 5^{\circ}$ of neutral, with the overall combined lower limb coronal orientation within $\pm 3^{\circ}$ of neutral. As discussed previously (see rKA, Chap. 17), in more complex cases requiring modification of the anatomy of both tibia and femur to stay within these limits, our practice is to preserve femoral anatomy as closely as possible and perform greater modifications on the tibial side. We believe that the femoral flexion axis plays the more significant role in knee kinematics. Femoral rotation is set at $0^{\circ}$ of rotation to the posterior condyles. The femoral size is matched to the best fit of the distal femoral anatomy, and sagittal orientation is set to avoid notching, usually at $2-4^{\circ}$ of flexion with respect to the mechanical axis of the femur. The tibial posterior slope is set at $3^{\circ}$ as recommended by the manufacturer. Application of the rKA protocol is performed by an experienced MyKnee ${ }^{\circledR}$ engineer at Medacta International. Then, the preoperative plan is sent to the surgeon according to these specifications for approval (Fig. 25.1). Images of the cuts and implants are simulated and provided (Fig. 25.2). The surgeon can modify the pre-operative plan if desired.

At the time of surgery, sterilised 3D bone models are provided with the PSI cutting guides (Fig. 25.3). Femoral and tibial cutting guides can be tested on the 3D bone models to assess the optimal fit (Fig. 25.4). As we use a CT-based 


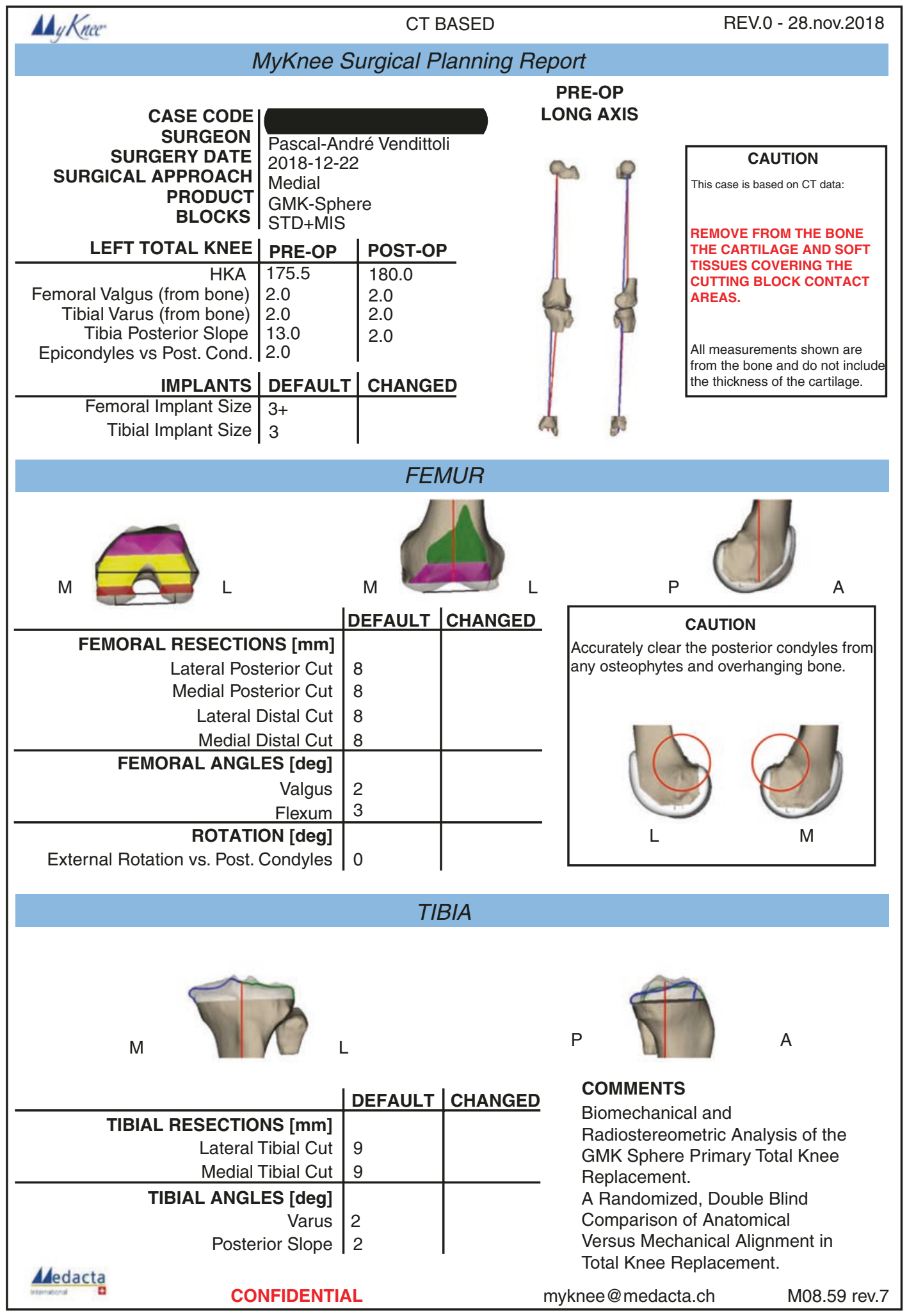

Fig. 25.1 Preoperative plan for rKA alignment 

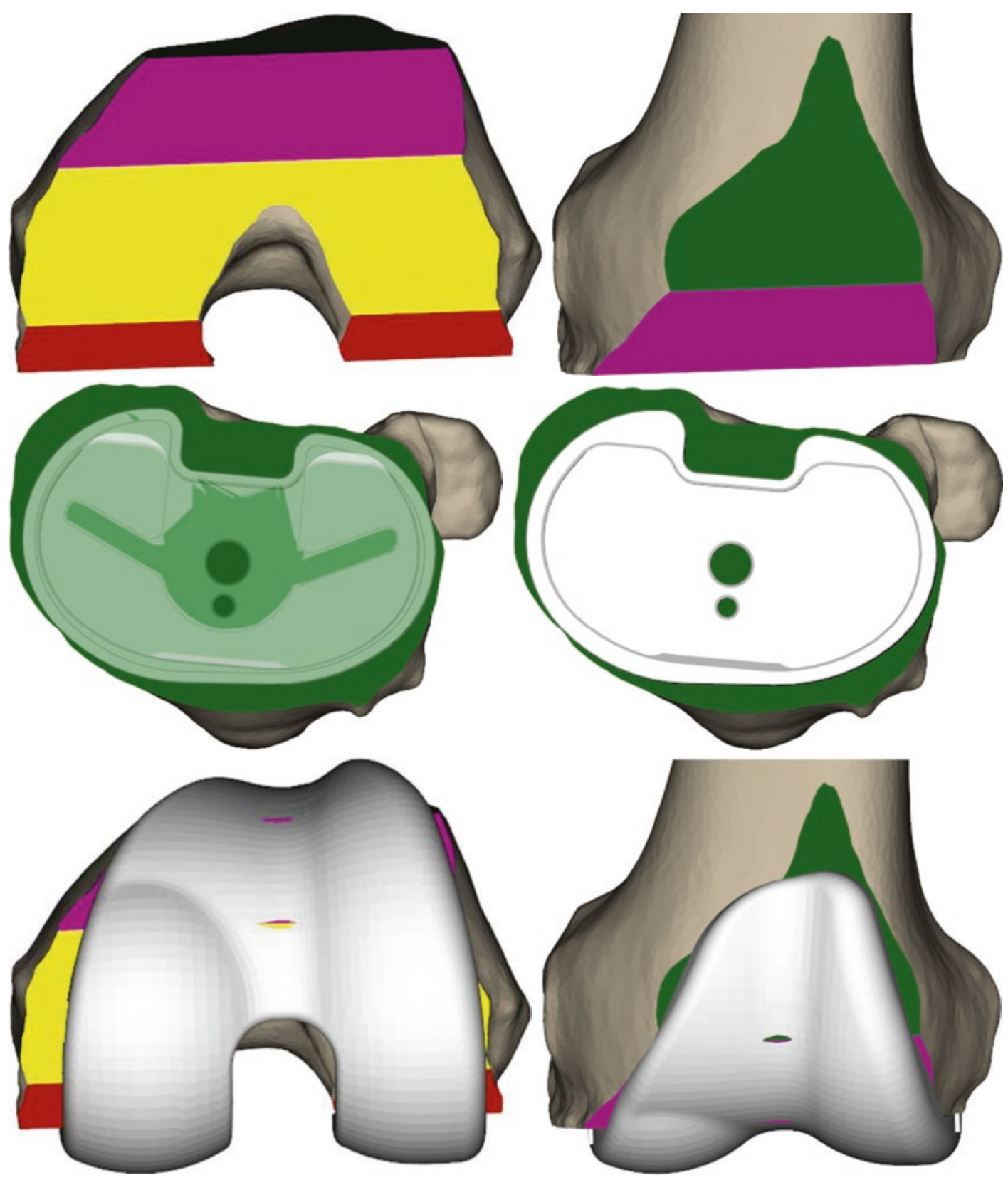

Fig. 25.2 Images of the resulting bone cuts with and without the implant are provided

protocol, the cartilage and soft tissues covering the cutting block contact areas must be removed from the bone with the help of a diathermy blade. The contact areas can be identified on the bone model (Fig. 25.2). The femoral cutting block is then placed manually on the distal femur, in the position of maximum stability (Fig. 25.5). Once the positioning is deemed satisfactory, the cutting block is fixed with pins. As well as positioning the guide for distal femoral resection, these pins set the rotation of the 4-in-1 cutting guide and hence femoral rotation.

For the tibial resection, the process is repeated with the tibial guide and bone model. To ensure 

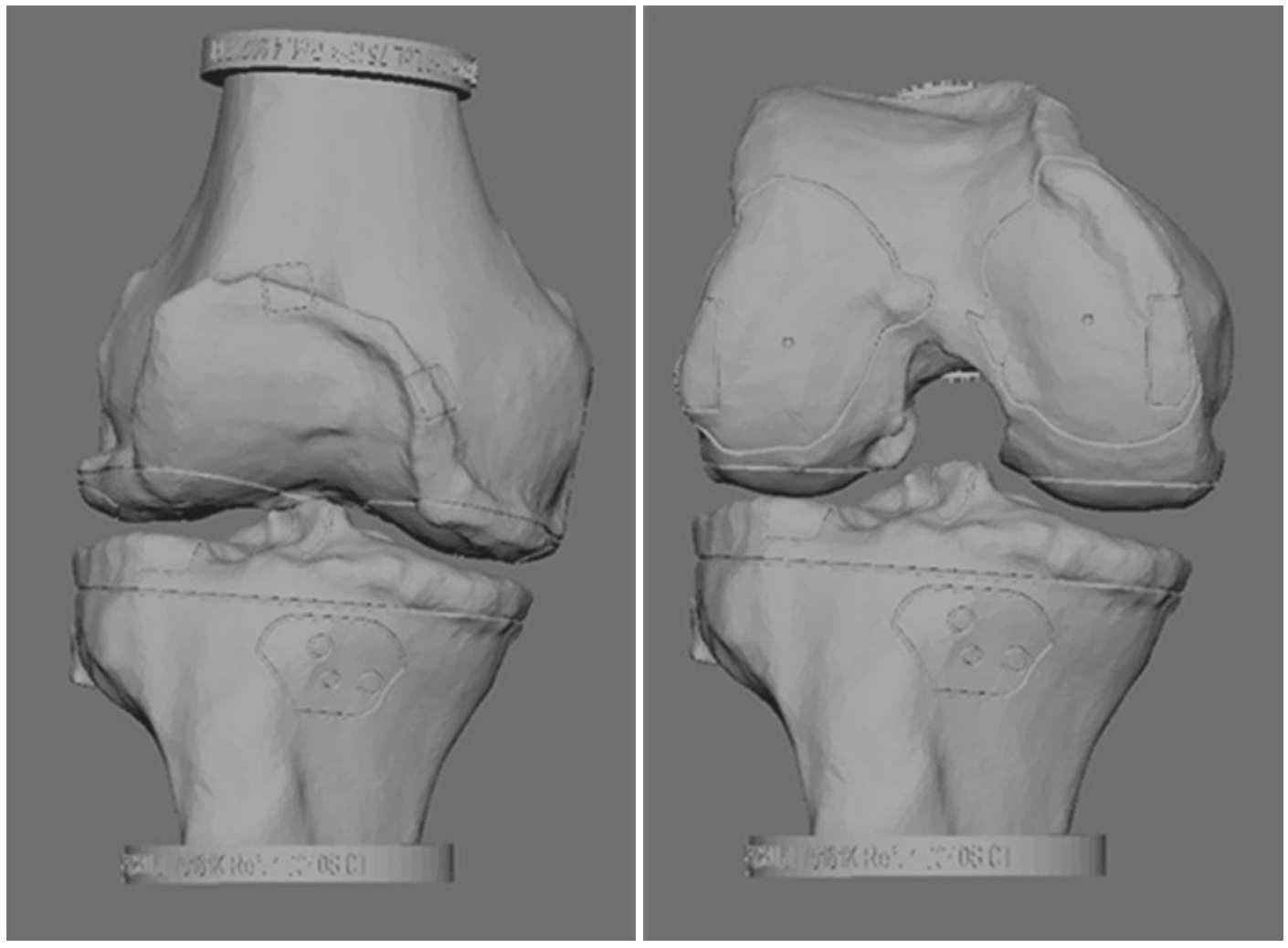

Fig. 25.3 3D bone models for an rKA case showing the equal medial and lateral cut thicknesses and the resulting joint line
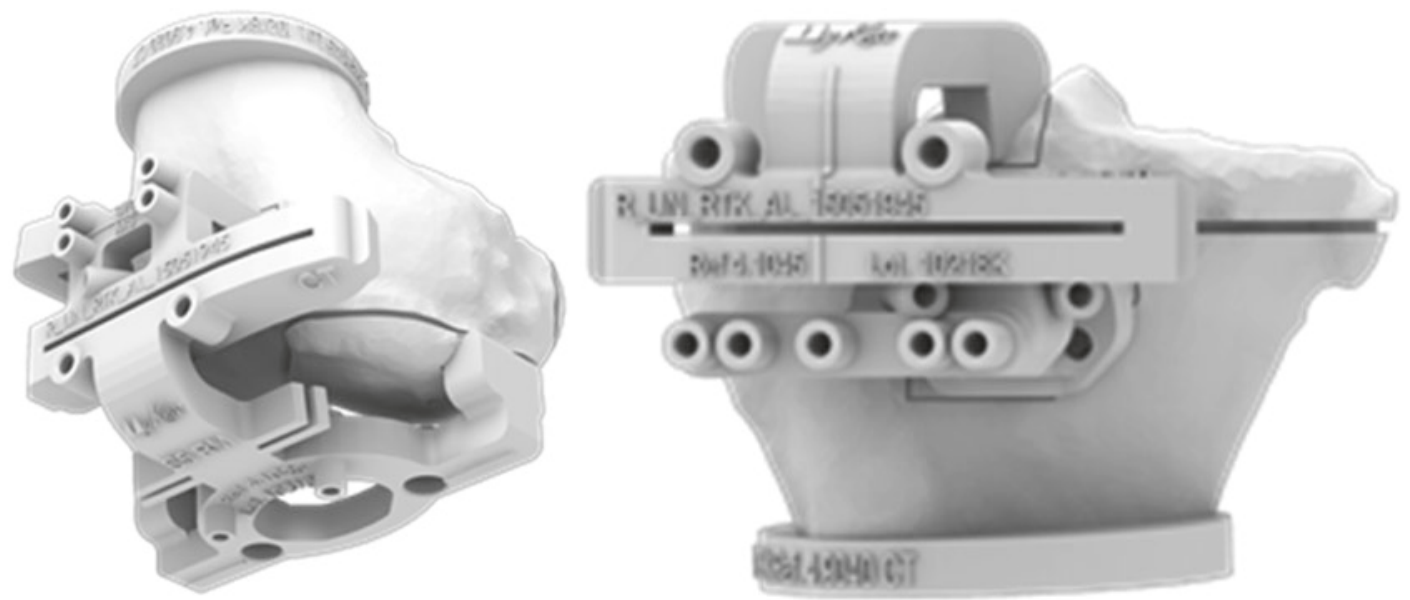

Fig. 25.4 Cutting blocks assembled on the 3D bone models to assess the optimal fit

maximum stability of the guide, the surgeon should verify that the points of contact between the tibial cutting block and the tibial bone correspond with the bone model (Fig. 25.4). Once the cutting guide has been properly positioned on the tibia, cut parameters are automatically set for the knee according to the preoperative plan (Fig. 25.6). With implant trials in place, 


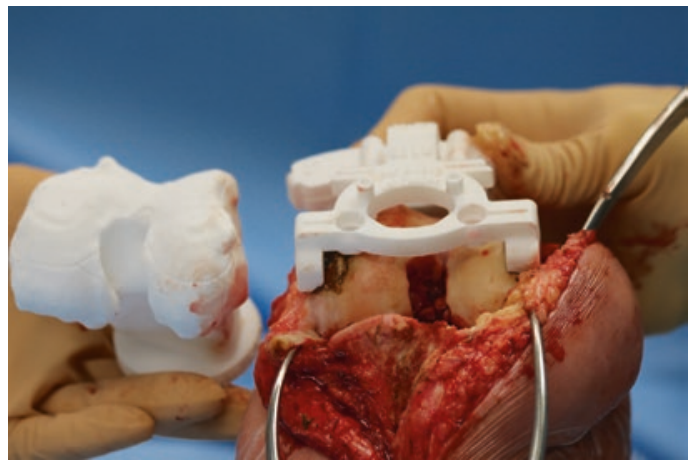

Fig. 25.5 Femoral block on the femur

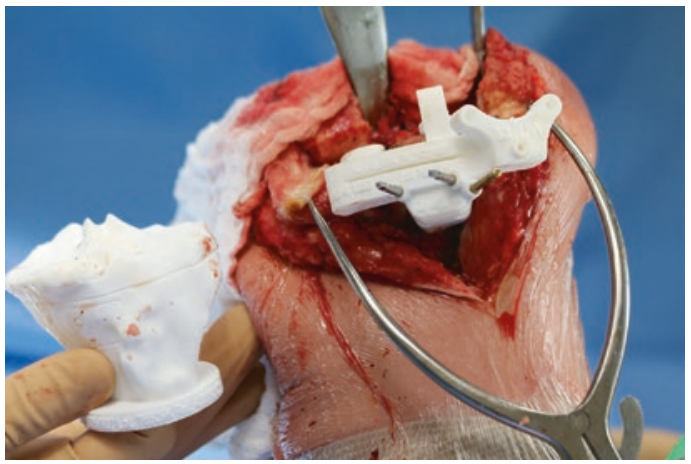

Fig. 25.6 Tibial block on bone

we adjust tibial rotation manually to match the femur in flexion and extension.

\subsection{Benefits of Patient-Specific Instruments}

The PSI method used by the authors (MyKnee ${ }^{\circledR}$, Medacta International SA, Castel San Pietro, Switzerland) has demonstrated accuracy of implant positioning in a number of studies [13-16]. A study of 50 consecutive TKAs performed using the MyKnee ${ }^{\circledR}$ PSI reported 98\% were within $3^{\circ}$ of the planned HKA angle [13]. Predicted coronal plane orientation of the tibial and femoral components was achieved in $100 \%$ and $96 \%$ of patients, respectively. The sagittal orientation of the femoral and tibial components was achieved in $98 \%$ and in $92 \%$ of patients, respectively. Accurate femoral rota- tion within $3^{\circ}$ of planned was accomplished in $90 \%$ of patients.

The majority of studies assessing PSI have demonstrated improved positioning of the femoral component compared to the tibia. It is the authors' experience that the femoral cutting guide is easier to position accurately due to the conforming anatomy of the distal femur. We would recommend that when positioning the tibia, the surgeon spends extra time to confirm accurate placement. Secondary checks may be performed with the alignment guides and following resection with callipers. In most cases of rKA TKA, no ligament imbalance is created. Using PSI for rKA thus simplifies the TKA procedure to a precise application of the cutting block on the patient's bone and avoidance of cut deviation using the oscillating saw. We prefer to use the Precision saw blade (Stryker, USA) for these procedures. This saw blade has an oscillating tip, but the core of the blade stays still. It eliminates blade vibration in the PSI cutting slot which avoids creation of plastic wear/debris.

Another benefit of PSI is standardisation of the procedure with all the planning done preoperatively, compared to computer navigation [11] or robotic surgery where it is done at the time of surgery. It is also rare to require recutting, in contrast to the calliper technique with conventional instrumentation (see Chap. 24). This may result in a shorter operating time. A meta-analysis demonstrated minor reductions in total operative time $(-4.4 \mathrm{~min}, p=0.002)$ and blood loss $(-37.9 \mathrm{~mL}, p=0.015)$ for PSI compared to conventional instrumentation [17]. The included studies all used mechanical alignment technique for implanting TKA. The time saved may be greater with a patient-specific alignment technique, where bespoke planning is required.

Other potential benefits include decreased instrumentation with less tray processing requirements and improved accuracy for the novice or low-volume surgeon (Fig. 25.7). A trial comparing PSI with conventional instrumentation demonstrates a 90-min reduction in instrument processing time [18]. With savings 
Fig. 25.7 Minimal instrumentation to perform TKA

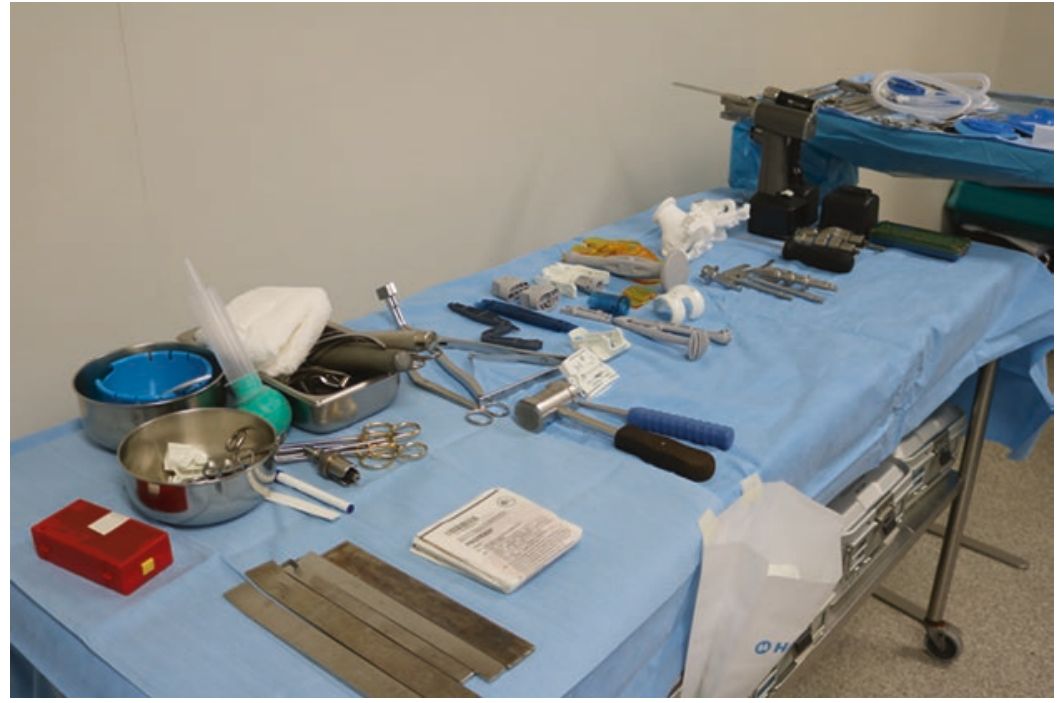

in labour, instrumentation and surgical time, this equated to a total savings of $\$ 628$ per case. They did note, however, that this was offset by the high cost of pre-operative imaging and fabrication of the PSI. These costs, however, are likely to be much less than those associated with robotic surgery, particularly in low-volume centres. Experienced surgeons are more accurate in their bone cuts using standard TKA instrumentation compared to surgeons with less experience [19]. Patient-specific instrumentation has been shown to improve accuracy of inexperienced surgeons to the equivalent of expert surgeons, in a study using sawbone knee models [20]. Most of the clinical studies comparing the accuracy of PSI with conventional instrumentation are conducted in high-volume centres with experienced arthroplasty surgeons [8] and may be subject to expert bias.

PSI is a simple, standardised solution for a patient-specific rKA protocol in TKA, with many benefits to the surgeon and patient.

\subsection{Case Example}

An active 58-year-old female, with advanced knee osteoarthritis, presents for consideration of TKA, after failure of conservative treatment. Six years ago, she had a right TKA in another institution with unsatisfying clinical results (pain and stiffness). Two years ago, she underwent a right TKA revision (by the initial surgeon) which did not improve her right knee function. She is severely disabled by her left knee OA but very hesitant to accept another TKA after the disappointing right knee results (Fig. 25.8). We offered her a left knee rKA TKA with PSI. A pre-operative planning CT scan demonstrates pre-operative femoral valgus of $2^{\circ}$ and tibial varus of $2^{\circ}$ (Figs. 25.1 and 25.2). The pre-operative HKA was $4.5^{\circ}$ varus, as a result of cartilage wear on the medial side of the tibia. The patient elected to undergo TKA using the rKA protocol. As is the case with $\sim 50 \%$ of cases, no modifications were required from her pre-operative constitutional alignment to stay within the safe range defined in our protocol, allowing a pure KA implantation. Frontal alignment was therefore $2^{\circ}$ valgus for the femoral component and $2^{\circ}$ varus for the tibial component, with an overall postoperative HKA of $0^{\circ}$. The patient underwent an uneventful post-operative recovery (Fig. 25.8b). At 4 months post-surgery, her prosthetic knee felt natural without restrictions. She is now requesting us to perform a second revision of her right knee to correct the implant orientations. 


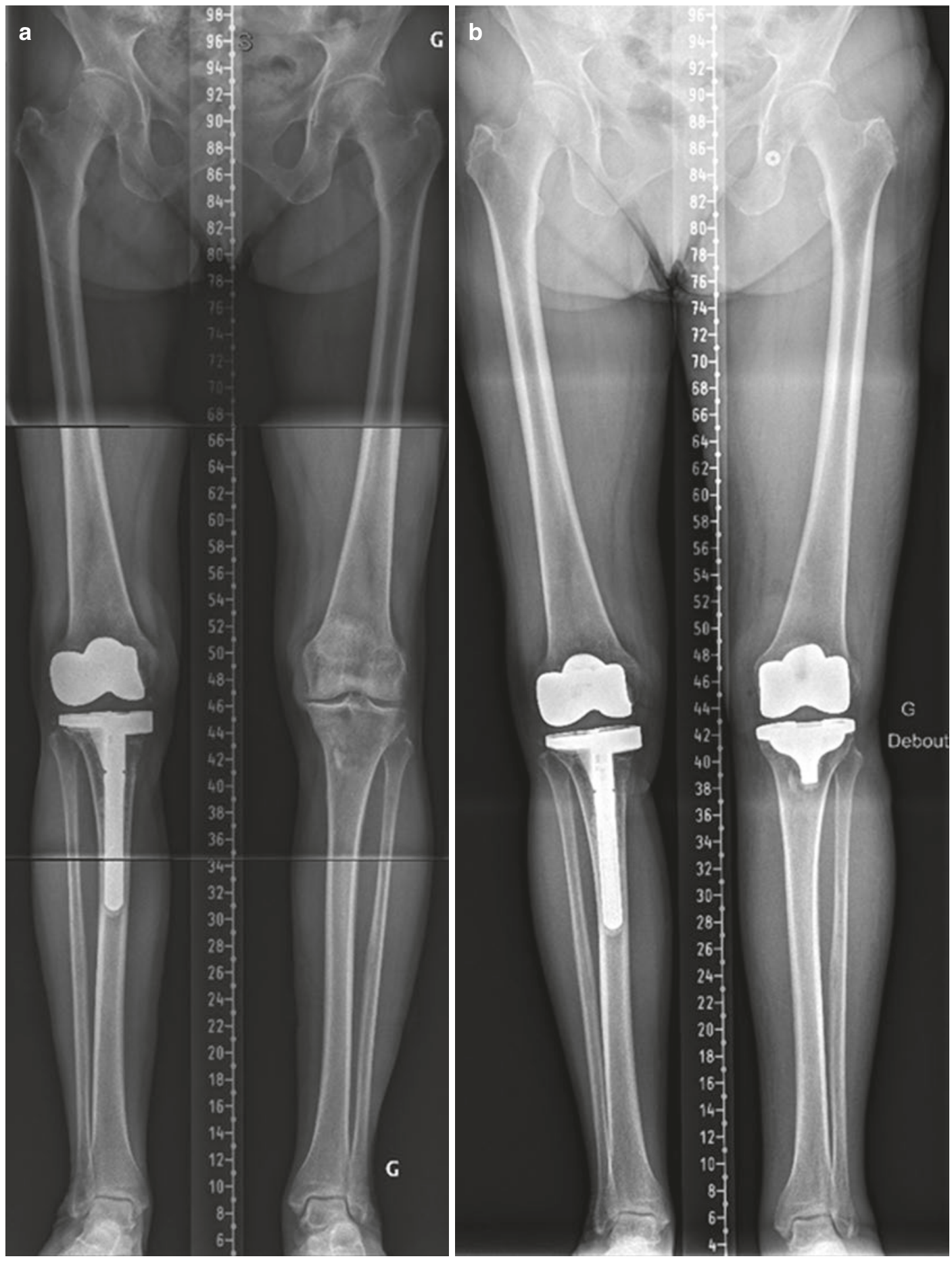

Fig. 25.8 Preoperative (a) and post-operative (b) standing long radiographs of the left knee rKA TKA with PSI. Patient unsatisfied with her right revision TKA (performed elsewhere) requested to be revised a second time 


\section{References}

1. Stirling P, Valsalan Mannambeth R, Soler A, Batta V, Malhotra RK, Kalairajah Y. Computerised tomography vs magnetic resonance imaging for modeling of patientspecific instrumentation in total knee arthroplasty. World J Orthop. 2015;6(2):290-7. Epub 2015/03/21

2. Fu H, Wang J, Zhou S, Cheng T, Zhang W, Wang $\mathrm{Q}$, et al. No difference in mechanical alignment and femoral component placement between patientspecific instrumentation and conventional instrumentation in TKA. Knee Surg Sports Traumatol Arthrosc. 2015;23(11):3288-95. Epub 17 Jul 2014

3. Jiang J, Kang X, Lin Q, Teng Y, An L, Ma J, et al. Accuracy of patient-specific instrumentation compared with conventional instrumentation in total knee arthroplasty. Orthopedics. 2015;38(4):e305-13. Epub 23 Apr 2015

4. Shen C, Tang ZH, Hu JZ, Zou GY, Xiao RC, Yan DX. Patient-specific instrumentation does not improve accuracy in total knee arthroplasty. Orthopedics. 2015;38(3):e178-88. Epub 12 Mar 2015

5. Zhang QM, Chen JY, Li H, Chai W, Ni M, Zhang ZD, et al. No evidence of superiority in reducing outliers of component alignment for patient-specific instrumentation for total knee arthroplasty: a systematic review. Orthop Surg. 2015;7(1):19-25. Epub 25 Feb 2015

6. Sharareh B, Schwarzkopf R. Review article: patientspecific versus standard instrumentation for total knee arthroplasty. J Orthop Surg (Hong Kong). 2015;23(1):100-6. Epub 30 Apr 2015

7. Cavaignac E, Pailhe R, Laumond G, Murgier J, Reina $\mathrm{N}$, Laffosse JM, et al. Evaluation of the accuracy of patient-specific cutting blocks for total knee arthroplasty: a meta-analysis. Int Orthop. 2015;39(8):154152. Epub 11 Oct 2014

8. Thienpont E, Schwab PE, Fennema P. A systematic review and meta-analysis of patient-specific instrumentation for improving alignment of the components in total knee replacement. Bone Joint J. 2014;96B(8):1052-61. Epub 3 Aug 2014

9. Ageberg E, Björkman A, Rosén B, Roos EM. Principles of brain plasticity in improving sensorimotor function of the knee and leg in patients with anterior cruciate ligament injury: a double-blind randomized exploratory trial. BMC Musculoskelet Disord. 2012; 13:68.
10. Mannan A, Akinyooye D, Hossain F. A metaanalysis of functional outcomes in patient-specific instrumented knee arthroplasty. J Knee Surg. 2017;30(7):668-74. Epub 3 Dec 2016

11. Hutt JRB, LeBlanc MA, Massé V, Lavigne M, Vendittoli PA. Kinematic TKA using navigation: surgical technique and initial results. Orthop Traumatol Surg Res. 2016;102(1):99-104.

12. Almaawi AM, Hutt JRB, Masse V, Lavigne M, Vendittoli P-A. The impact of mechanical and restricted kinematic alignment on knee anatomy in total knee arthroplasty. J Arthroplast. 2017;32(7):2133-40.

13. Nabavi A, Olwill CM, Do M, Wanasawage $T$, Harris IA. Patient-specific instrumentation for total knee arthroplasty. J Orthop Surg (Hong Kong). 2017;25(1):2309499016684754. Epub 1 Feb 2017

14. Lyras DN, Greenhow R, Loucks C. Restoration of the mechanical axis in total knee artrhoplasty using patient-matched technology cutting blocks. A retrospective study of 132 cases. Arch Bone Joint Surg. 2017;5(5):283-9. Epub 12 Dec 2017

15. Koch PP, Muller D, Pisan M, Fucentese SF. Radiographic accuracy in TKA with a CT-based patient-specific cutting block technique. Knee Surg Sports Traumatol Arthrosc. 2013;21(10):2200-5. Epub 15 Aug 2013

16. Anderl W, Pauzenberger L, Kolblinger R, Kiesselbach G, Brandl G, Laky B, et al. Patient-specific instrumentation improved mechanical alignment, while early clinical outcome was comparable to conventional instrumentation in TKA. Knee Surg Sports Traumatol Arthrosc. 2016;24(1):102-11. Epub 20 Oct 2014

17. Thienpont E, Schwab PE, Fennema P. Efficacy of patient-specific instruments in total knee arthroplasty: a systematic review and meta-analysis. J Bone Joint Surg Am. 2017;99(6):521-30. EpuB 16 Mar 2017

18. Barrack RL, Ruh EL, Williams BM, Ford AD, Foreman K, Nunley RM. Patient specific cutting blocks are currently of no proven value. J Bone Joint Surg Br. 2012;94(11 Suppl A):95-9. Epub 9 Nov 2012

19. Plaskos C, Hodgson AJ, Inkpen K, McGraw RW. Bone cutting errors in total knee arthroplasty. J Arthroplast. 2002;17(6):698-705. Epub 7 Sept 2002

20. Jones GG, Logishetty K, Clarke S, Collins R, Jaere M, Harris S, et al. Do patient-specific instruments (PSI) for UKA allow non-expert surgeons to achieve the same saw cut accuracy as expert surgeons? Arch Orthop Trauma Surg. 2018;138:1601-8. Epub 5 Sept 2018

Open Access This chapter is licensed under the terms of the Creative Commons Attribution 4.0 International License (http://creativecommons.org/licenses/by/4.0/), which permits use, sharing, adaptation, distribution and reproduction in any medium or format, as long as you give appropriate credit to the original author(s) and the source, provide a link to the Creative Commons license and indicate if changes were made.

The images or other third party material in this chapter are included in the chapter's Creative Commons license, unless indicated otherwise in a credit line to the material. If material is not included in the chapter's Creative Commons license and your intended use is not permitted by statutory regulation or exceeds the permitted use, you will need to obtain permission directly from the copyright holder. 